\title{
The Atmosphere of Venus: A Conference Review
}

\author{
Francis S. Jomsson \\ Southwest Center for Advanced Studies, Dallas, Tex.
}

(Manuscript received 5 April 1968)

\begin{abstract}
Data from Mariner 5, the Soviet planetary probe Venera 4, and recent ground-based measurements have provided much new information and permitted a great step forward in our knowledge of Venus. Although the data from the various sources generally complement one another very well to provide a fairly detailed picture of the atmosphere of Venus, several serious discrepancies exist that still require clarification. These relate to composition, particularly oxygen and water, and to the surface pressure and temperature. On the positive side, it is now clear that the atmosphere is very dry, with great pressure and high temperature at the surface. It also appears increasingly probable that the greenhouse theory can account for the very high surface temperature. The most likely explanation for the clouds is that they are of convective type consisting of ice crystals and with tops near the 0.2 -atm pressure level.
\end{abstract}

\section{Introduction}

The descent stage of the Soviet spacecraft, Venera 4, has provided the first direct measurements in the atmosphere of Venus. These measurements are nicely supplemented by measurements made from the American spacecraft, Mariner 5, as it flew past the planet. Further, ground-based measurements provide additional valuable information that permits the development of a reasonably detailed picture of the atmosphere of Venus, probably more detailed than can be developed for Mars at this time.

\section{Surface temperature and pressure}

The Soviet measurements (Nikiforoff, 1967; Davydov, 1968; Vakhnin, 1968; Avduevsky et al., 1968 ) indicated a nearly dry adiabatic lapse rate between the pressure levels 18.5 and $0.72 \mathrm{~atm}$, where the temperatures were $544 \mathrm{~K}$ and $298 \mathrm{~K}$, respectively. The composition was observed to be mainly carbon dioxide (approximately $90 \%$ or higher), and the atmosphere was very dry, less than $0.7 \%$ water vapor (Vinogradov et al., 1968). The upper level of measurement was identified as occurring at an altitude of $26 \mathrm{~km}$; this determination was made by a radio altimeter which gave a repetitive response indicating that the descent stage was either above or below an altitude of $26 \mathrm{~km}$, and it changed from indicating above $26 \mathrm{~km}$ to below $26 \mathrm{~km}$ just after the parachute on the instrument capsule opened. The last point of observation, where the pressure was $18.5 \mathrm{~atm}$, was deduced to be at the surface on the basis of integration downward of the barometric equation from the $26-\mathrm{km}$ level indicated by the radio altimeter, and also from the calculated rate of fall for the parachute from that level; if the observations were not actually terminated at the surface, then it is a coincidence that the signals failed at just that altitude where the surface should have been expected to be on the basis of the radio altimeter indication at the beginning of the measurement period.

The indication from Venera 4 that the surface pressure is $18.5 \mathrm{~atm}$ is in disagreement with the Mariner 5 observations when they are combined with radar data on the radius of Venus. For example, the 0.72 -atm pressure level was found from Mariner 5 to lie at a planetocentric distance of $6114 \mathrm{~km}$ on the nightside and 6109 $\mathrm{km}$ on the dayside (Kliore et al., 1967). The radar radius of the planet is $6056 \mathrm{~km}$ (Ash et al., 1967), indicating an altitude of $58 \mathrm{~km}$ for the 0.72 -atm pressure level on the nightside, whereas the Soviet figure for the altitude of this pressure level is $26 \mathrm{~km}$. Thus, there is a discrepancy of $32 \mathrm{~km}$ between the altitude scales determined by the Soviet radio altimeter (and by the termination of transmissions from the entry capsule) and the Mariner 5 trajectory data combined with radar data on the radius of Venus. Uncertainties in the time of closest approach change the Mariner scale somewhat, and the distance scale on the nightside has been decreased by about $7 \mathrm{~km}$ (Kliore and Cain, 1968), decreasing the discrepancy to $25 \mathrm{~km}$. However, this adjustment of the altitude scale should also apply to the dayside of the planet, but with opposite sign. Thus, the 0.72 -atm pressure level, instead of being $5 \mathrm{~km}$ higher on the nightside than on the dayside as indicated above, apparently is now indicated to be $9 \mathrm{~km}$ higher on the dayside than on the nightside, a difference that is unacceptable from the standpoint of atmospheric physics. From this viewpoint, the $7-\mathrm{km}$ correction in altitude scale is an overcorrection; $2.5 \mathrm{~km}$ should be close to the correct value unless there is an inconsistancy between the dayside and nightside scales presented by Kliore et al. (1967). Further, new data on the radar radius indicate a still smaller value, $6050 \mathrm{~km}$, as most probable (Ash et al., 
1968); this tends to increase the discrepancy, so it may be as large as $35 \mathrm{~km}$. However, this number is likely to change somewhat with further trajectory analysis.

It does not appear likely that the difference in altitude scales can be explained by the landing of the Soviet capsule on a mountain or other high ground. The required elevation appears unreasonable, especially in view of the high surface temperature and the probable existence of near molten conditions not far below the surface.

If the surface of Venus is actually some $35 \mathrm{~km}$ below the point where the last Soviet measurement was made, the surface pressure must be far in excess of the 18.5 atm measured in Venera 4 , and the temperature probably considerably in excess of $544 \mathrm{~K}$. This could provide a better agreement with the microwave emission spectrum from the planet. The microwave emission cannot be completely accounted for by a surface temperature as cool as $544 \mathrm{~K}$; it fits better with a temperature of 650 or $700 \mathrm{~K}$ (Kuzmin and Vetukhnovskaya, 1968). If the adiabatic lapse rate were to continue below the last Soviet measurement, a temperature of $700 \mathrm{~K}$ would be reached about $17 \mathrm{~km}$ below that last measurement, and the pressure there would be about $65 \mathrm{~atm}$. If the surface were $35 \mathrm{~km}$ below the last Soviet measurement and the lapse rate remained adiabatic all the way to the surface, the surface temperature would be above $800 \mathrm{~K}$ and the surface pressure would be about $200 \mathrm{~atm}$. However, the adiabatic lapse rate might change to a stable distribution before reaching the surface, so that the temperature at the surface might not exceed $700 \mathrm{~K}$ even if it were $35 \mathrm{~km}$ below the last Soviet measurement.

Thus, the surface pressure and temperature remain in doubt. The Soviet results are $18.5 \mathrm{~atm}$ and $544 \mathrm{~K}$, but the Mariner 5 results, combined with radar data, indicate that this particular pressure level and temperature occur at an altitude near $35 \mathrm{~km}$. The surface pressure and temperature, therefore, may be in excess of $100 \mathrm{~atm}$ and $700 \mathrm{~K}$.

\section{Tropospheric lapse rate}

The temperature distribution above the highest Soviet measurement can be expected to remain dry adiabatic for some distance, because the air is very dry. Two phosphorus pentoxide electrically conductive detectors indicated moisture content in excess of $0.1 \%$ and $0.05 \%$, whereas a calcium chloride detector indicated less than $0.7 \%$ (Vinogradov et al., 1968). At 298K and $0.72 \mathrm{~atm}$, the moisture content would have had to be near $4.5 \%$ to produce saturation; thus, the Soviet observations indicate that the relative humidity was only a few percent at that pressure altitude. Therefore, if mixing were to prevail up to the top of the cloud or haze layer where the temperature is $230 \mathrm{~K}$, the dry adiabatic lapse rate would extend upward to the 0.2atm level. Even if the atmosphere contained the maximum moisture permitted by the Soviet observations
$(0.7 \%)$, the deviation from a dry adiabatic lapse rate would be very small. The cloud tops may therefore be expected to occur at an altitude of $34 \mathrm{~km}$ on the Soviet scale or a planetocentric distance of $6115 \mathrm{~km}$ on the Mariner 5 scale (Kliore and Cain, 1968), or $6122 \mathrm{~km}$ according to the scale indicated by Kliore et al. (1967). If a less steep temperature lapse rate than dry adiabatic were to prevail near the cloud layer, the cloud tops would have to occur at a lesser pressure than 0.2 $\mathrm{atm}$, or at a higher temperature than $230 \mathrm{~K}$. The spectroscopic data of $0.2 \mathrm{~atm}$ and $230 \mathrm{~K}$ that are now generally accepted as describing conditions at the cloud tops are not compatible with the Venera 4 data and anything very different from a dry adiabatic lapse rate up to the cloud tops. This is in apparent mild disagreement with the occulation data, which seem to indicate a transition region between the adiabatic region and the stratosphere, with the 0.2 -atm pressure level falling somewhat near the middle of the transition region. However, the possibility of a double tropopause near the latitude of occulation, $37 \mathrm{~N}$, similar to that which occurs on earth at similar latitudes, confuses the situation in such a way that it is difficult to assert that the disagreement is significant.

\section{Cloud composition}

a. Ice crystals

The most reasonable expectation for the composition of the cloud particles appears to be ice crystals. The presence of 1) water vapor in excess of $0.1 \%, 2$ ) convection as indicated by the extensive region with a nearly dry adiabatic lapse rate, and 3) temperatures near $230 \mathrm{~K}$ at the top of the cloud or haze layer, collectively argue for ice crystals. The strongest argument against ice crystals is the low water vapor content observed spectroscopically (Kuiper ${ }^{1}$ ); this indicates a water vapor mixing ratio near $10^{-6}$ above or in the upper portion of the haze layer. Such a figure can be reconciled with the Soviet figure $\left(>10^{-3}\right)$ only if the temperature near the top of the haze layer is as low as about $190 \mathrm{~K}$. Although such a suggestion sounds a little extreme, it is not necessarily unreasonable, and it possibly provides a means of reconciling the spectroscopic data with the Soviet direct measurements. One might visualize cumulus towers reaching to the 190K level, the cloud density being greatest at the cloud tops, but still relatively thin by comparison with cumulus clouds on earth (the ice crystal content of the cloud being of the order of a few tenths of a gram per cubic meter). The tropopause should be near the cloud tops, and the air above the tropopause might be very dry, similar to the situation above the earth's tropopause where the water vapor mixing ratio is as low as about $10^{-6}$, apparently as a result of passing through a cold trap of about $190 \mathrm{~K}$ at

\footnotetext{
${ }^{1}$ Kuiper, G. P., 1968: Paper presented at the Second Arizona Conference on Planetary Atmospheres, Tucson, 11-13 March.
} 
the tropical tropopause. Under such conditions, it is not entirely clear what the spectral observations should indicate; appropriate model calculations should be made to see if the spectroscopic observations are consistent with a dry stratosphere above cold cloud tops. A fairly thin cold layer near the tropopause, with a tropopause temperature of $190 \mathrm{~K}$, might not be recognizable in the infrared measurements. Actually, the existence of limb darkening in the infrared (Chase et al., 1963) indicates the presence of temperatures lower than the $230 \mathrm{~K}$ bolometric temperature not far above the cloud tops.

\section{b. Chemical compounds}

Connes et al. (1967) have observed $6 \times 10^{-7} \mathrm{HCl}$ and $10^{-8} \mathrm{HF}$ in the atmosphere of Venus. This has led to speculation that the clouds might consist of chloride compounds. Ammonium chloride might appear to be a likely candidate, but ammonia has not been detected at the $5 \times 10^{-8}$ level $\left(\right.$ Benedict $\left.^{2}\right)$. Lewis ${ }^{3}$ has drawn attention to the possibility that mercury compounds, including $\mathrm{Hg}_{2} \mathrm{Cl}_{2}$, might contribute to the clouds, because mercury should be released and vaporized at the surface as a result of the high temperatures there.

\section{c. Dust}

Dust has also been suggested for the clouds on Venus. Squires, ${ }^{4}$ however, has presented powerful arguments that the cloud formation should involve the release of latent heat, tending to favor the concept of ice clouds produced by convective activity. It is hard to imagine how clouds of dust could be raised on Venus, as the massive atmosphere must restrict winds near the surface to low velocities. The heat capacity is so great that diurnal variations cannot exceed about $10 \mathrm{~K}$ at most, perhaps only a $1-2 \mathrm{~K}$, even though the length of the Cytherean day is very long, 117 earth days. Shower clouds on earth produce wind gusts that raise dust, but these are not to be expected on Venus. Probably the most reasonable mechanism that might inject dust into the atmosphere is volcanic activity. However, it seems improbable that dust clouds in any case could produce the observed high albedo.

\section{Temperature above cloud levels}

Above the cloud tops, the temperature must be approximately constant up to the $10^{-3}$ atm pressure level, as indicated by the $5.4-\mathrm{km}$ scale height observed at the commencement of occultation on Mariner 5 (Kliore and Cain, 1968). For a molecular weight appropriate to carbon dioxide, the temperature should be about $245 \mathrm{~K}$ at the $10^{-3} \mathrm{~atm}$ pressure level, which may be significantly higher than that at the cloud tops (a situation

2 Benedict, W. A., 1968. Paper presented at the Second Arizona Conference on Planetary Atmospheres, Tucson, 11-13 March.

${ }^{3}$ Lewis, J., 1968: Paper presented at the Second Arizona Conference on Planetary Atmospheres, Tucson, 11-13 March.

4 Squires, P., 1968: Paper presented at the Second Arizona Conference on Planetary Atmospheres, Tucson, 11-13 March. similar to the warm upper stratosphere at the corresponding pressure level on earth). The $10^{-3} \mathrm{~atm}$ pressure level occurs near a geocentric distance of about $6144 \mathrm{~km}$. At higher levels, the best estimate of temperature is provided by the theoretical calculations of McElroy (1968), the upper portion agreeing well with the temperatures deduced from the hydrogen corona observations of Barth (1968). A sketch of the probable temperature and particle concentration distribution is shown in Fig. 1. The temperature below $6080 \mathrm{~km}$ is uncertain, and plausible distributions are shown by the dashed lines. The possibility of a temperature as low as $190 \mathrm{~K}$ at the cloud tops is indicated by a dotted line.

\section{Composition}

\section{a. Water vapor}

Reference has already been made to the water vapor question, where the Soviet measurements indicate several parts per thousand, while the spectroscopic data indicate about one part per million. Although this apparent discrepancy may possibly be explained by a dry stratosphere model, a clear resolution of the question is required. Observations from above of cold cloud tops penetrating the tropopause on earth might contribute useful information.

The lack of water on Venus remains a very puzzling question; if it were present originally and lost through dissociation and hydrogen escape, massive oxidation of the surface would have been required, something that cannot at present be disproved but which seems unlikely. Possibly free carbon may have been present in sufficient quantities to convert the water to carbon dioxide by the water gas reaction, which proceeds readily at the temperatures existing on Venus. This lack of water is probably the major geochemical question concerning Venus.

\section{b. Oxygen}

The second major question of composition in the Cytherean atmosphere concerns oxygen. The Soviet measurements indicate over four parts per thousand, as indicated by the burnout of an $800 \mathrm{~K}$ tungsten filament (Vinogradov et al., 1968), whereas spectroscopic data indicate less than 40 parts per million (Belton et al., 1968). Inasmuch as no effects are known that might change the proportion of oxygen in the atmosphere at these pressure levels, it will be difficult to accept the Soviet result unless it is confirmed by repeated measurement.

\section{c. Nitrogen}

It is probable that a few per cent nitrogen may exist in the atmosphere of Venus; this is a reasonable expectation by comparison with the development of the atmosphere on earth, and such a relative concentration has been suggested for Mars (Johnson, 1965). 


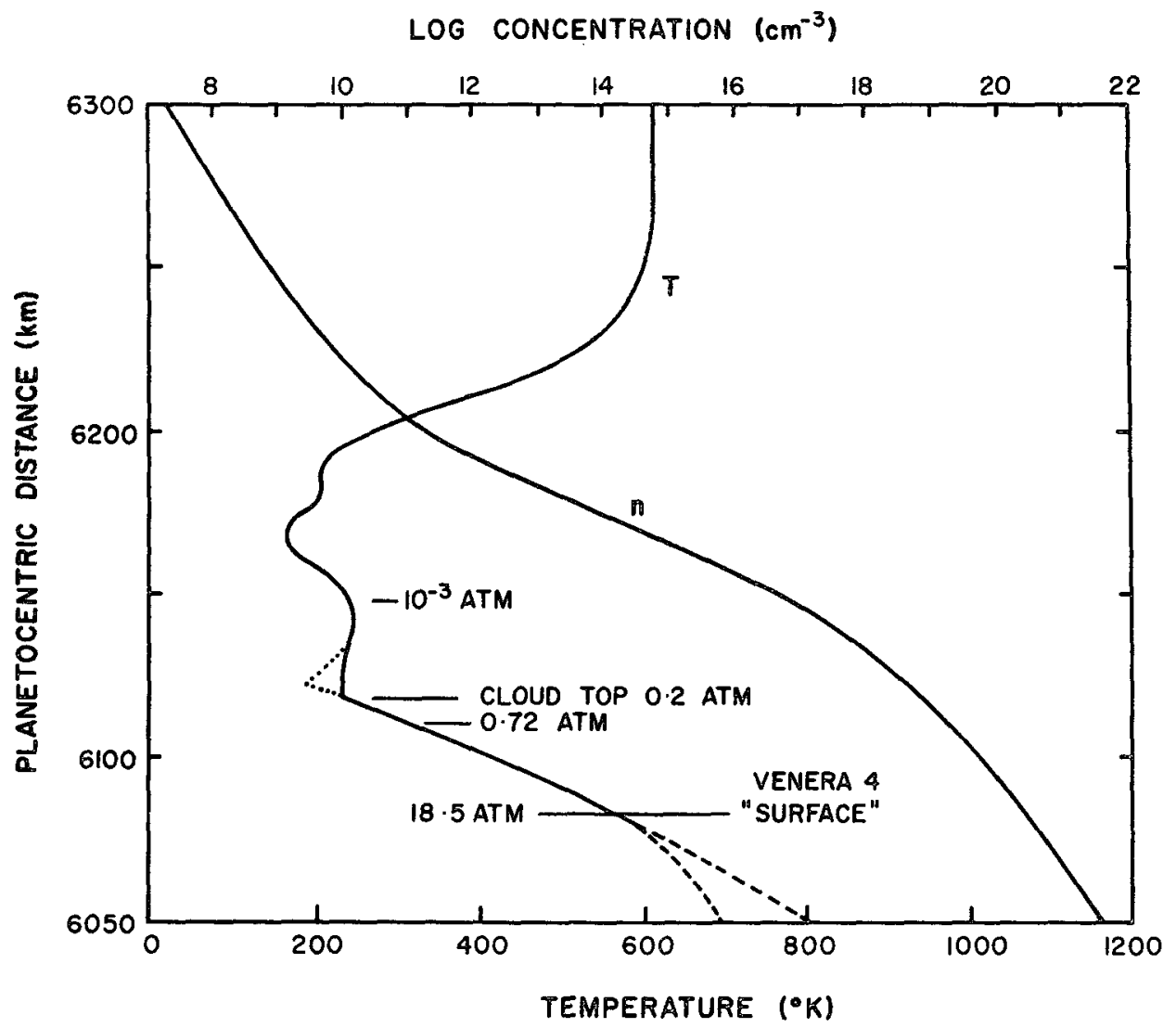

FIG. 1. A summary view of the atmosphere of Venus. Temperature and $\mathrm{CO}_{2}$ concentrations are shown as functions of altitude. The region from 18.5 to $0.72 \mathrm{~atm}$ is based upon Venera 4 measurements, but on a planetocentric distance scale adjusted to fit Mariner 5 occultation data. From 0.72 to $10^{-3} \mathrm{~atm}$, the distribution is based on Mariner 5 data but modified to agree with atmospheric structure concepts associated with cloud physics. At levels above the $10^{-3}$ atm pressure level, the distribution is in general agreement with the calculations of McElroy. The dotted portion of the temperature curve suggests a condition with cloud top temperatures near $190 \mathrm{~K}$, whereas the solid curve indicates $230 \mathrm{~K}$. The dashed curves suggest possible temperature distributions below the lowest Soviet measurement, supposing that the Soviet probe did not actually reach the surface. Radar data indicate that the surface is located near the bottom of the figure.

\section{Hydrogen escape}

The problem of water and hydrogen escape leads one to the hydrogen corona, which also possesses a puzzling feature; namely, the rapid increase in Lyman alpha luminosity near the planet on the sunlit side. The attempt to explain this in terms of molecular hydrogen which produces Lyman alpha radiation after undergoing a photodissociation (Barth, 1968) does not appear to be one that will be seriously regarded for very long, as the recombination of atomic species into molecular probably cannot be accomplished at the required rate. The problem of explaining it in terms of a two-temperature distribution also appears most difficult. In some respects, it seems more attractive to assume that the lower part of the hydrogen cloud consists mainly of deuterium, assuming that the deuterium-to-hydrogen ratio has been enriched by fractionation brought about by thermal escape. This explanation also suffers a major defect in that it is difficult to imagine that thermal escape prevails over other escape mechanisms on a planet like Venus that possesses no significant intrinsic magnetic field. The main escape mechanism is probably the ionization of atmospheric particles at high altitudes (greater than $500 \mathrm{~km}$ ) and their subsequent acceleration away into interplanetary space by the electric fields associated with the solar wind; this loss mechanism would not favor hydrogen over deuterium beyond that degree associated with their differential distributions under conditions of diffusive equilibrium, a degree which appears entirely inadequate to account for the observations.

The atomic hydrogen concentrations around Venus are significantly less than those around earth at comparable distances. Kurt's ${ }^{5}$ observations from Venera 4 indicate $50 \mathrm{~cm}^{-3}$ at a distance of $10,000 \mathrm{~km}$ from the planet's center on the nightside, whereas Barth's data on the dayside indicate a larger value, about $200 \mathrm{~cm}^{-3}$.

${ }^{5}$ Reported by A. D. Kuzmin at the Second Arizona Conference on Planetary Atmospheres, Tucson, 11-13 March. 
However, hydrogen concentrations at this distance from the earth vary in the range $10^{3}-10^{4}$, depending upon the phase of the solar cycle. A water vapor content of: $10^{-6}$ above the clouds on Venus is an adequate source for the hydrogen through photodissociation; photodissociation of $\mathrm{HCl}$ is another possibility.

\section{Induced magnetosphere}

The interaction of the solar wind with Venus presents another problem. Cloutier et al. ${ }^{6}$ described an interaction of the solar wind with the Cytherean atmosphere that results in a deviation of the flow of the solar wind around the planet as a result of a buildup of particle pressure. A different view of the interaction to be expected was put forth earlier by Dessler (1967), in which he indicated that the ionospheric conductivity would result in a current flow and a magnetic field buildup above the ionosphere of sufficient strength to deviate the solar wind. Dessler did not discuss the variation of magnetic field strength as a function of altitude. It is possible that the field buildup extends over a region of several hundred kilometers on the dayside and constitutes an induced magnetosphere, and that its outer boundary coincides with the plasmapause observed by the Mariner Stanford Group (1967). Below this plasmapause or magnetopause, the ionosphere is protected from direct influence of the solar wind, and above the magnetopause, any ions formed from the ambient atmosphere are rapidly swept away by the solar wind. According to this concept, the ionosphere produced in an atmosphere by solar ultraviolet radiation causes the development of an induced magnetosphere that offers considerable protection to the atmosphere against direct bombardment by the solar wind and limits loss of atmospheric particles by solar wind interactions to rather modest values, perhaps a few tens of meters of gas at STP over geologic time.

Acknowledgments. This work was supported by National Aeronautics and Space Administration under grant NsG-269.

${ }^{6}$ Cloutier, P., M. B. McElroy and F. C. Michel, 1968: Paper presented at Second Arizona Conference on Planetary Atmospheres, Tucson, 11-13 March.

\section{REFERENCES}

Avduevsky, V. S., M. Ya. Marov and M. K. Rozhdestvensky, 1968: Model of the atmosphere of the planet Venus based on results of measurements made by the Soviet automatic interplanetary station Venera 4. J. Atmos. Sci., 25, 537-545.

Ash, M. E., I. I. Shapiro and W. B. Smith, 1967: Astronomical constants and planetary ephemerides deduced from radar and optical observations. Astron. J., 72, 338-350.

- $e t$ al., 1968: The case for the radar radius of Venus. J. Atmos. Sci., 25, 560-563.

Barth, C. A., 1968: Interpretation of the Mariner 5 Lyman alpha measurements. $J$. Atmos. Sci., 25, 564-567.

Belton, M. J. S., A, Lyle Broadfoot and D. M. Hunten, 1968: Upper limit to $\mathrm{O}_{2}$ on Venus. $J$. Atmos. Sci., 25, p. 582.

Chase, S. C., L. D. Kaplan and G. Neugebauer, 1963: The Mariner 2 infrared radiometer experiment. J. Geophys. Res., 68, 6157-6169.

Connes, P., J. Connes, W. S. Benedict and L. D. Kaplan, 1967: Traces of $\mathrm{HCl}$ and $\mathrm{HF}$ in the atmosphere of Venus. A strophys. $J ., 147,1230-1237$.

Davydov, V. D., 1968: New view of Venus. Intern. Sci. Tech., No. $75,49-54$.

Dessler, A. J., 1968: Ionizing plasma flux in the Martian upper atmosphere. Atmospheres of Venus and Mars, London, Gordon and Breach.

Johnson, F. S., 1965: Atmosphere of Mars. Science, 150, $1445-1448$.

Kliore, A. and D. L. Cain, 1968: Mariner 5 and the radius of Venus. J. Atmos. Sci., 25, 549-554.

—_, G. S. Levy, D. L. Cain, G. Fjeldbo and S. I. Rasool, 1967 : Atmosphere and ionosphere of Venus from the Mariner V S-band radio occultation measurement. Science, 158, 16831688.

Kuzmin, A. D., and Yu. N. Vetukhnovskaya, 1968: Venera 4 and the interpretation of the radio astronomical measurements of Venus. $J$. Atmos. Sci., 25, 546-548.

Mariner Stanford Group, 1967: Venus: Ionosphere and atmosphere as measured by dual-frequency radio occultation of Mariner V. Science, 158, 1678-1683.

McElroy, M. B., 1968: The upper atmosphere of Venus. $J$. Geophys. Res., 73, 1513-1521.

Nikiforoff, C. C., 1967: Venus 4: An automatic interplanetary station. Trans. Amer. Geophys. Union, 48, 931-937.

Vakhnin, V. M., 1968: A review of the Venera 4 flight and its scientific program. J. Atmos. Sci., 25, 533-534.

Vinogradov, A. P., U. A. Surkov and C. P. Florensky, 1968: The chemical composition of the Venus atmosphere based on the data of the interplanetary station Venera 4. J. Atmos. Sci., 25, 535-536. 